Supplement of J. Sens. Sens. Syst., 8, 261-267, 2019

https://doi.org/10.5194/jsss-8-261-2019-supplement

(C) Author(s) 2019. This work is distributed under

the Creative Commons Attribution 4.0 License.

(c) (1)

Supplement of

\title{
Enabling a new method of dynamic field-effect gas sensor operation through lithium-doped tungsten oxide
}

Marius Rodner et al.

Correspondence to: Tilman Sauerwald (t.sauerwald@lmt.uni-saarland.de)

The copyright of individual parts of the supplement might differ from the CC BY 4.0 License. 


\section{S1 Characterization methods}

Characterization of the film morphology was done with atomic force microscopy (AFM) and scanning electron microscopy (SEM) whereas X-ray diffraction (XRD) and Raman measurements were performed to gain information about the crystalline structure and the size of the crystallites within the layers.

An AFM (Veeco Dimension 3100) with a silicon cantilever and a lateral resolution of $1 \mathrm{~nm}$ was used in tapping mode with a scanning frequency of $1 \mathrm{~Hz}$. The overall roughness $R_{q}$ in height is calculated for a random spot within the measurement using the formula for the root mean square deviation (RMSD):

$$
R_{q}=\sqrt{\frac{\sum_{i=1}^{N}\left(Z_{i}-Z_{\text {average }}\right)^{2}}{N}}
$$

Here, $N$ is the number of points, $Z_{i}$ the height of each point and $Z_{\text {average }}$ the average of all heights. Before characterization with the SEM (Zeiss Sigma FESEM), all samples were coated with a $5 \mathrm{~nm}$ layer of sputtered platinum to ensure good conductance and achieve the maximum resolution. To gain a crosssection view, the backside of each sample is scribed with a laser and then cracked. Within the SEM measurement, the setup provides the possibility of performing EDX measurements. For a cross-section view of the complex FET structures a dual beam FIB (FEI Helios DualBeam) was used to cut a $1 \mu \mathrm{m}$ deep channel before taking an SEM image. Before cutting a channel into the layer, a $0.5 \mu \mathrm{m}$ thick protective platinum layer was deposited on top of the desired cutting spot. Both deposition and cutting were performed in serpentine mode. The Raman spectra were acquired with a HORIBA Jobin Yvon LabRAM HR800 using 2D and 3D filters and a light source with a wavelength of $488 \mathrm{~nm}$. Wave numbers between $50 \mathrm{~cm}^{-1}$ and $900 \mathrm{~cm}^{-1}$ were used to determine the characteristic peaks of different $\mathrm{WO}_{3}$ phases. Differences observed between $50 \mathrm{~cm}^{-1}$ and $500 \mathrm{~cm}^{-1}$ allow differentiation between $\mathrm{WO}_{3}$ epsilon and gamma phases, whereas differences observed at higher wave numbers can also be attributed to rotations of tungsten to oxygen bonds. The XRD (Bruker D8 Discover with Da Vinci design) was used with grazing incidence diffraction (GID) at a $2 \Theta$-angle between $11^{\circ}$ and $52^{\circ}$, so that peaks from the silicon in the SiC substrate are suppressed.

\section{S2 Additional sample characterizations}

Figure S1 shows the SEM cross sections together with an AFM measurement of its surface as inset. The layer clearly shows a column-like structure with small particles and a very flat surface and exhibits an average roughness $R_{q}$ of $2.99 \mathrm{~nm}$.

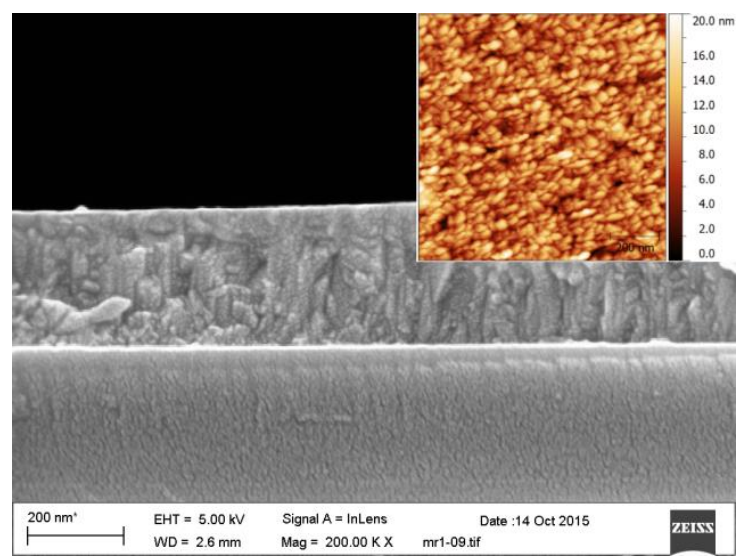

Figure S1: $\quad$ SEM cross-sections with inset showing an AFM scan over an area of $1 \times 1 \mu \mathrm{m}^{2}$. 
The XRD patterns in Fig. S2 show the difference in crystalline orientation for the different tungsten oxide layers. For the as-deposited (RT) sample (dashed blue line), an amorphous phase with no distinct orientation can be seen, which changes to a highly orientated phase when in-situ annealing instead (solid black line) with clear peaks between $23^{\circ}$ and $33^{\circ}$ in the 20 -angle diagram (Cazzanelli et al., 1999; Johansson et al., 2012). The small contribution around $23^{\circ}$ is most likely a mixture of the $\varepsilon$-phase (002) reflection at $2 \theta=22.95^{\circ}$ and the $\gamma$-phase (002) orientation at $23.15^{\circ}$ (Johansson et al., 2012; Righettoni et al., 2010). The major reflections of the in-situ annealed film are located at angles $2 \theta=28.5^{\circ}$ and $33.2^{\circ}$. Distinguishing between the $\gamma$-phase and $\varepsilon$-phase from the different reflections in this angle range is difficult because of the similarity of the crystal structures, which are both monoclinic and very similar in size, and, thus, the XRD reflections are also very close to each other. XRD measurements determine a particle size of $24 \mathrm{~nm}$ using integral breadth method.

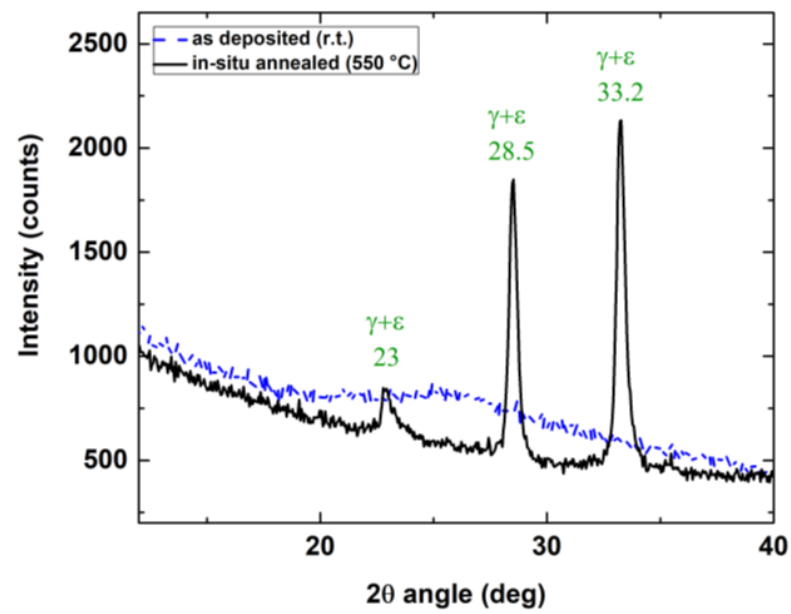

Figure S2: $\quad$ XRD spectra for undoped $\mathrm{WO}_{3}$ layers deposited at room temperature (blue, dashed) and at $550{ }^{\circ} \mathrm{C}$ (black, solid).

\section{S3 Additional sensor measurements}

Similar to the measurements shown in Fig. 3 (b) and 4, two others sensors from a second batch have been tested to show the reproducibility of the observed effects. In Fig. S3, the drain current $\mathrm{I}_{\mathrm{D}}$ is shown while altering the gate voltage between $-2,0$ and $+2 \mathrm{~V}$. For the negative bias, the drain current decreases almost to zero as the electron channel is almost closed. The time constants of this sensor pair is much smaller and the overshoot at a higher positive voltage of $+2 \mathrm{~V}$ is not as high as for the results shown in Fig. 3 (b), but again, the overshoot, followed by a relaxation effect can only be seen for the doped sensor (blue line). 


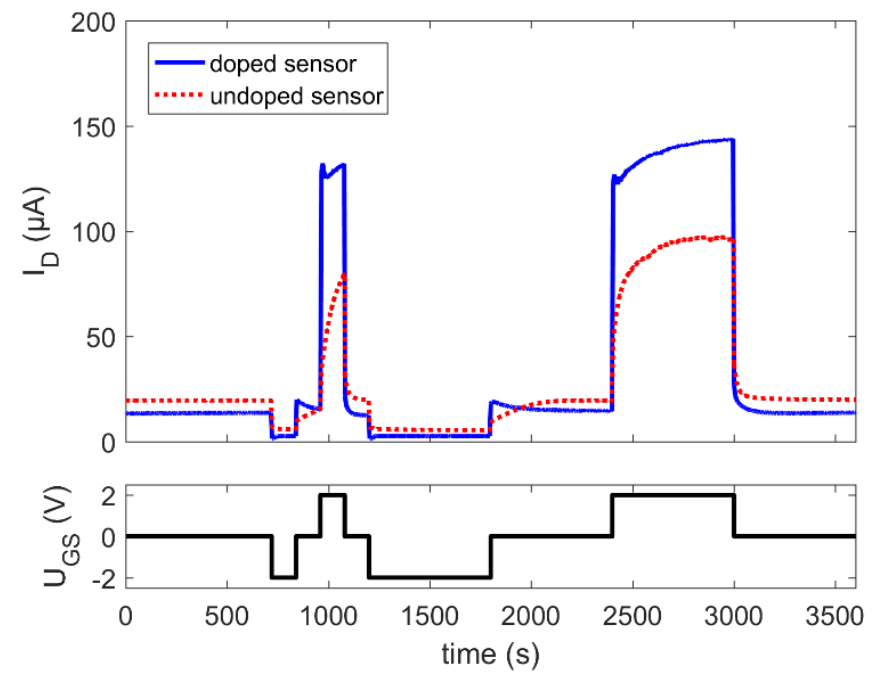

Figure S3: $\quad$ Drain current $I_{D}$ at different gate voltages for doped and undoped samples. The different gate potentials $-2,0$ and $+2 \mathrm{~V}$ are shown in the lower subplot.

This second pair of sensors was then exposed to 50 and $100 \mathrm{ppm} \mathrm{NH}$ at $-2,0$ and $+2 \mathrm{~V}$ gate voltage. For $-2 \mathrm{~V}$, no sensor response can be seen as the drain current $\mathrm{I}_{\mathrm{D}}$ is almost completely depleted to zero and therefore the response is not shown in Fig. S4. The response of the undoped sensor is almost not influenced by the gate bias whereas a large increase in response can be found for the doped sensor when increasing the gate voltage from 0 to $+2 \mathrm{~V}$. Similarly to the results shown in Fig. 4 , also the sensitivity increases only for the doped sensor.

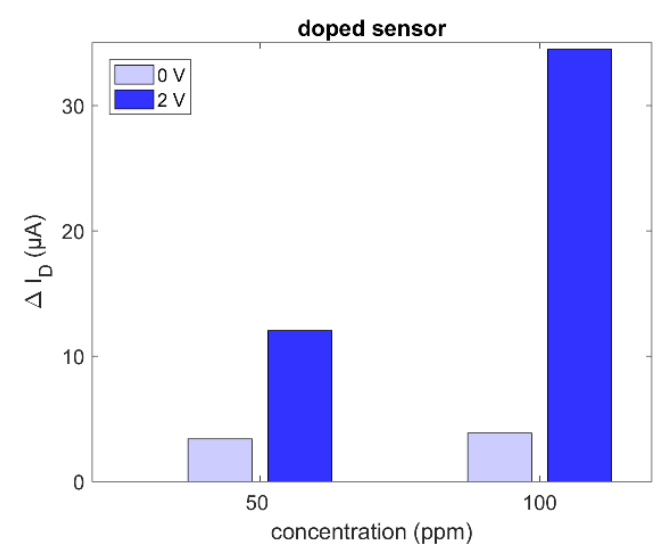

(a)

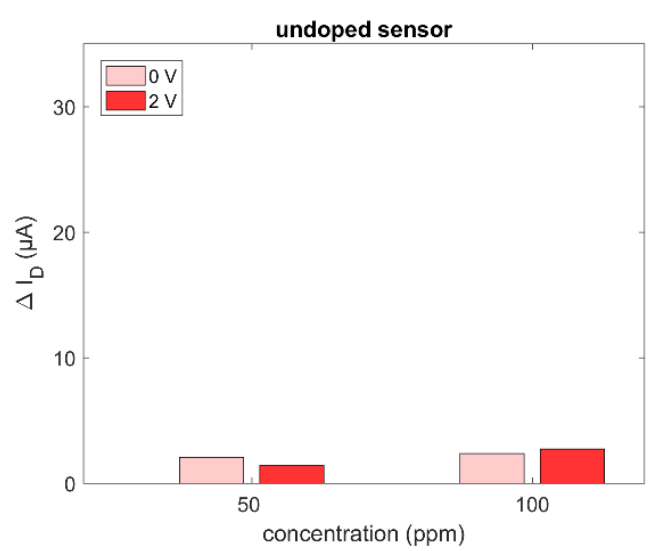

(b)

Figure S4: $\quad$ Sensor response at gate voltages of 0 and $2 \mathrm{~V}$ for doped (a) and undoped (b) sensor at 50 and 100 ppm $\mathrm{NH}_{3}$.

\section{References}

Cazzanelli, E., Vinegoni, C., Mariotto, G., Kuzmin, A. and Purans, J.: Low-Temperature Polymorphism in Tungsten Trioxide Powders and Its Dependence on Mechanical Treatments, J. Solid State Chem., 143(1), 24-32, doi:10.1006/jssc.1998.8061, 1999.

Johansson, M. B., Niklasson, G. a. and Österlund, L.: Structural and optical properties of visible active photocatalytic WO3 thin films prepared by reactive dc magnetron sputtering, J. Mater. Res., 27(24), 3130-3140, doi:10.1557/jmr.2012.384, 2012.

Righettoni, M., Tricoli, A. and Pratsinis, S. E.: Thermally stable, silica-doped $\varepsilon$-WO3 for sensing of acetone in the human breath, Chem. Mater., 22(10), 3152-3157, doi:10.1021/cm1001576, 2010. 